\title{
Complete Neck Clipping of Internal Carotid-Posterior Communicating Artery Aneurysms Using Bayonet-Shaped Aneurysm Clips
}

\author{
-Technical Note-
}

\author{
Hiroshi Kashimura, Kuniaki OgasawARA, Yoshitaka KuBO, and Akira OgAWA
}

Department of Neurosurgery, Iwate Medical University School of Medicine, Morioka, Iwate

\begin{abstract}
Neck clipping for internal carotid-posterior communicating artery (IC-PC) aneurysms using standard straight, angled, or curved clip may result in remnant aneurysm neck. We describe complete neck clipping of IC-PC aneurysms using a bayonet-shaped clip. The bayonet-shaped clip is applied perpendicular to the long axis of the internal carotid artery (ICA), and the blades of the clip are inserted between the aneurysm neck and the ICA. Using the clip applicator, the clip is gradually rotated counterclockwise or clockwise for left or right ICA aneurysm, respectively, so that the distal and shank portions of the clip blade are located at the aneurysm neck in the posterior communicating artery (Pcom) and ICA, respectively. As a result, the distal flexure of the clip blade fits the junction of the ICA and Pcom. This technique was used in four patients with ruptured ICA aneurysms and five patients with unruptured ICA aneurysms. Postoperative cerebral angiography demonstrated no residual aneurysm neck and preservation of the Pcom in all patients. This technique is useful for cases of IC-PC aneurysm involving the origin of the Pcom.
\end{abstract}

Key words: cerebral aneurysm, clipping, internal carotid artery, bayonet-shaped clip

\section{Introduction}

Aneurysms arising from the internal carotid artery (ICA) are common, ${ }^{4)}$ and are usually located at the junction of the ICA and posterior communicating artery (Pcom). These aneurysm necks frequently involve the origin of the Pcom (Fig. 1). Neck clipping for internal carotid-posterior communicating artery (IC-PC) aneurysms using the usual straight, angled clip or curved clip may result in a remnant aneurysm neck. Here we describe complete neck clipping of IC-PC aneurysms using a bayonet-shaped clip.

\section{Surgical Technique}

The bayonet-shaped clip has a flexure in the two portions of the blades, forming proximal, shank, and distal portions (Fig. 2). After the proximal sylvian cistern is opened toward the ICA bifurcation, the artery proximal to the lesion is exposed for proximal

Received October 11, 2006; Accepted March 19, 2007

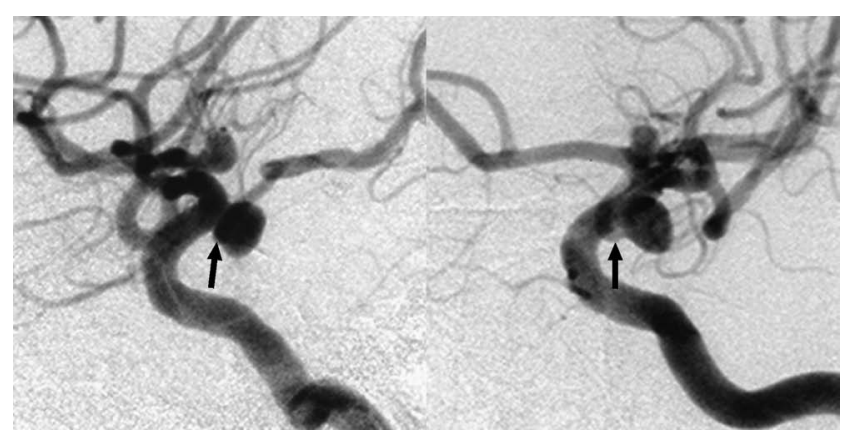

Fig. 1 Lateral and oblique left carotid angiograms in a 60-year-old woman with unruptured aneurysms of the left internal carotidposterior communicating artery and $A_{1}$ segment of the anterior cerebral artery. Angiography shows the posterior communicating artery arising from the proximal side of the aneurysm neck (arrow).

control. The origin and course of the Pcom, branches of the Pcom, and anterior choroidal artery are confirmed (Fig. 3A), and the aneurysm dome is fully exposed. First, the bayonet-shaped clip is applied 


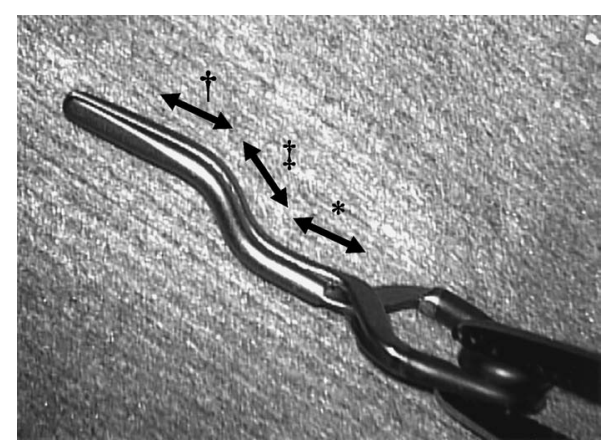

Fig. 2 Photograph of a bayonet-shaped Yasargil titanium clip (No. FT727T). The clip has a flexure in the two portions of the blades, forming proximal $(*)$, shank $(\ddagger)$, and distal portions ( $\dagger$ ).

perpendicular to the long axis of the ICA, and the blades of the clip are inserted between the aneurysm neck and ICA (Fig. 3B). Using the clip applicator, the clip is gradually rotated counterclockwise or clockwise for left or right ICA aneurysm, respectively, so that the distal and shank portions of the clip blade are located at the aneurysm neck in the Pcom and ICA, respectively (Fig. 3C). As a result, the distal flexure of the clip blade fits the junction of the ICA and Pcom (Fig. 3C). Preservation of branches arising from the Pcom and absence of residual aneurysm neck are then confirmed (Fig. 3D).

We have used this technique for four patients with ruptured and five patients with unruptured IC-PC aneurysms. No patients experienced postoperative neurological deficits and postoperative computed tomography showed no new ischemic lesions in any case. In addition, postoperative cerebral angiography demonstrated no residual aneurysm neck and confirmed preservation of the Pcom in all patients (Fig. 4).

\section{Discussion}

Although the distal flexure and shank portions of the bayonet clip were not originally designed for occluding the aneurysm itself,1) the present technique is useful for cases of IC-PC aneurysms in which the neck is broad and involves the Pcom. When distal and shank portions of the clip blade fit the aneurysm neck in the Pcom and ICA, respectively, the origin of the Pcom can be reconstructed.

This technique is unsuitable for IC-PC aneurysms under the following conditions: Large aneurysms, as the length of the shank portion may be too short for the aneurysm neck and the length of the shank portion is not variable; if the ICA is laterally tortu-
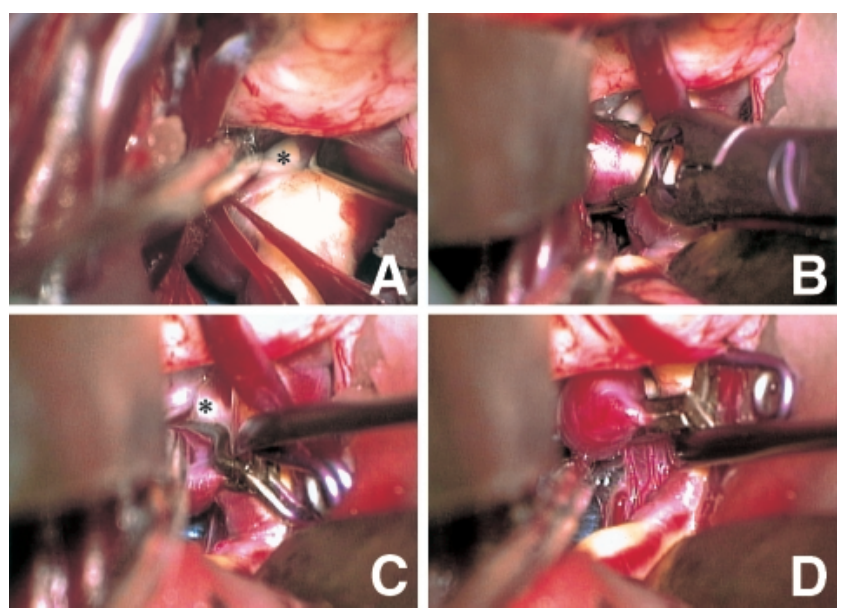

Fig. 3 Intraoperative photographs in a 60-year-old woman with unruptured aneurysms of the left internal carotid-posterior communicating artery and $A_{1}$ segment of the anterior cerebral artery. A: Origin of the posterior communicating artery (Pcom) (*) was confirmed after exposing the internal carotid artery (ICA) for proximal control. B: A bayonet-shaped Yasargil titanium clip (No. FT727T) was applied perpendicular to the long axis of the ICA, and the blades were gradually inserted between the aneurysm neck and ICA. C: The distal flexure of the clip blade fitted the junction of the ICA and Pcom. The origin of the Pcom (*) was reconstructed with the bayonet-shaped clip. D: Preservation of branches arising from the Pcom and absence of residual aneurysm neck are confirmed.

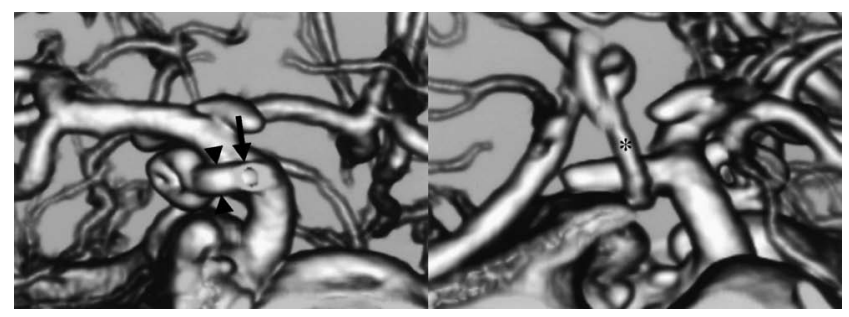

Fig. 4 Postoperative posteroanterior and medial computed tomography angiograms showing complete neck clipping of the left internal carotid-posterior communicating artery aneurysm and aneurysm of the $A_{1}$ segment of the anterior cerebral artery. The distal flexure (arrow) and shank portion (arrowheads) of the bayonet-shaped clip fitted the left internal carotid artery. The posterior communicating artery (*) was preserved and no residual aneurysm neck was present. 
ous, as the clip blades of the distal portion may touch the posterior clinoid process or ipsilateral oculomotor nerve; if the aneurysm projects ventrally, as standard clipping with fenestrated clips is recommended $^{2,3)}$; and if severe sclerotic changes are present in the junction between the ICA and Pcom, as the risk of Pcom stenosis or occlusion may increase. Confirmation of Pcom flow using Doppler ultrasonography is recommended.

\section{Acknowledgments}

This work was supported in part by Grants-in-Aid for Advanced Medical Science Research from the Ministry of Education, Culture, Sports, Science and Technology, Japan (2004-2008).

\section{References}

1) Osawa M, Obinata C, Kobayashi S, Tanaka Y: Newly designed bayonet clips for complicated aneurysms: technical note. Neurosurgery 36: 425-427, 1995

2) Sugita K, Kobayashi S, Inoue T, Banno T: New angled fenestrated clips for fusiform vertebral artery aneurysms. J Neurosurg 54: 346-350, 1981

3) Sugita K, Kobayashi S, Kyoshima K, Nakagawa F: Fenestrated clips for unusual aneurysms of the carotid artery. J Neurosurg 57: 240-246, 1982

4) Yasargil MG: Pathological considerations, in Yasargil MG, Smith RD, Young PH, Teddy PJ (eds): Microneurosurgery I. Microsurgical Anatomy of the Basal Cisterns and Vessels of the Brain, Diagnostic Studies, General Operative Techniques and Pathological Considerations of the Intracranial Aneurysms. Stuttgart, Georg-Thieme-Verlag, 1984, pp 299-301

Address reprint requests to: Hiroshi Kashimura, M.D., Department of Neurosurgery, Iwate Medical University School of Medicine, 19-1 Uchimaru, Morioka, Iwate 020-8505, Japan.

e-mail: hkashi@iwate-med.ac.jp

\section{Commentary}

The authors should be congratulated on bringing up this important technical note regarding the shape of the clip.

It is well known that the best positioning of the clip is along the longitudinal axis of the artery (ICA), since in this way, the least narrowing of the diameter of the artery results. The next general principle in clipping of an aneurysm is that the aneurysm should be excluded completely (no rest of the aneurysm after positioning the clip) and that the daughter arteries should be preserved open. This is easy to say, but sometimes difficult to achieve. The quality of the wall of the artery (ICA), the size of the neck (of the aneurysm), the proximity of the PCom as well as the size of the aneurysm, all play an important role in the whole maneuver of exclusion of an aneurysm from the circulation. The technical note of using bayonetshaped aneurysm clips is important as a reminder for the surgeon that such a clip does exist on his table, and that the surgeon shouldn't be satisfied just with the exclusion of the aneurysm, but should pay attention also to how the aneurysm is excluded. We all know that most aneurysms would require an individually designed clip; however, this is impossible, and that's why a compromise should be made on the above mentioned requirements regarding morphology of the artery with aneurysm, daughter arteries, as well as according to the completeness of the exclusion of the aneurysm itself.

This report brings the message that we may achieve better and long lasting results in aneurysm exclusion, if we are aware of the existing possibilities that are already at hand and are available. I am not advocating by this the numerous changing of different clips, in order to find an appropriate one for an "angry" aneurysm. My intention is to remind the surgeon with not so much experience in aneurysm surgery to think twice before the decision on the shape and size of the clip is made.

Vinko V. Dolenc, M.D. Department of Neurosurgery University Medical Center Ljubljana, Slovenia

The authors describe the technical considerations of bayonet-shaped aneurysm clips for relatively broad based internal carotid artery-posterior communicating artery (IC-PC) aneurysms.

In the era of developing endovascular surgery for aneurysm obliteration, long-term durability of the procedure is essentially important to select either neck clipping or coil embolization, which is determined by individual requirements. One of the weak points of the endovascular technique is strictly complete occlusion of broad neck aneurysms, even though some supportive procedures such as the balloon assisted technique have been developed. The IC-PC aneurysm, the most common site of cerebral aneurysms, often has wide aneurysm neck toward the origin of the posterior communicating artery and complete obliteration of such an aneurysm is not always easy. Although the original purpose of the bayonet-shaped aneurysm clip was to reduce the dead angle caused by the clip applicator around clip blades in deep and narrow operating fields, successfully applied bayonet-shaped clip as described in this technical note can avoid both complicated clipping procedures using multiple clips and residual aneurysm neck.

Yoshiaki SHIOKawa, M.D. Department of Neurosurgery Kyorin University School of Medicine Mitaka, Tokyo, Japan 